\title{
A Non-Uniform-Angular-Rate Beam-Scan Method for Airborne TOPS Mode
}

\author{
Yifu GUAN, Wenge CHANG \\ College of Electronic Science and Engineering, National University of Defense Technology, \\ No. 109 Deya Road, Kaifu District, Changsha, Hunan Province, 410073, P. R. China \\ guanyifu9@sina.com
}

Submitted December 5, 2017 / Accepted May 9, 2018

\begin{abstract}
TOPS (Terrain Observation by Progressive Scans) mode can achieve large scene coverage through the beam-scan in a uniform-angular-rate form; when the scanning angle is large, the variation of azimuth theoretical resolution with the scanning angle cannot be ignored. Aiming at airborne SAR (Synthetic Aperture Radar) system, the Doppler characteristic of azimuthal echo of the TOPS mode with large scanning angle is analyzed, and a non-uniform-angular-rate beam-scan method is proposed. By adjusting the angular rate of the beam-scan in a real-time form, we can achieve a consistent azimuth theoretical resolution for the targets at different azimuth location. The simulation results show that the proposed method can alleviate the azimuth resolution inconsistency of about $0.4 \mathrm{~m}$ when the azimuth theoretical resolution is $5 \mathrm{~m}$.
\end{abstract}

\section{Keywords}

TOPS, non-uniform, beam-scan, airborne, SAR

\section{Introduction}

Airborne digital array SAR has the characteristics of flexible beam pointing. It can be combined with digital beam-forming technology to realize simultaneous multimode imaging, which is one of the research hotspots of airborne SAR [1-3].

The TOPS mode was originally proposed as a spaceborne wide-swath imaging mode to overcome the scalloping phenomenon in ScanSAR [4] and has been used in the European Space Agency (ESA) Sentinel -1 and the German Aerospace Center (DLR) TerraSAR-X [5]. In order to meet the requirement of airborne SAR to cover large imaging area within a short working period, TOPS mode was later used on the airborne SAR platform [6], [7].

When working in TOPS mode, the Doppler parameters (Doppler centroid frequency and Doppler modulation rate) of the target and the dwell time of the beam on the target are functions of the beam scanning angle, which results in the inconsistent azimuth theoretical resolution for the targets at different azimuth location. For spaceborne TOPS mode, due to its small scanning angle, the issue above might be ignored based on the principle of smallangle-approximation [8]. However, for the airborne TOPS mode, the scanning angle is much larger than that in the spaceborne TOPS mode in order to obtain sufficient azimuth coverage of the scene, and the small-angle-approximation is no longer appropriate. Therefore, when dealing with airborne TOPS mode, especially with large scanning angle, the inconsistent azimuth theoretical resolution is an issue that we must consider.

When processing the echo data of TOPS mode, the algorithms based on the sub-aperture method can handle the issue of inconsistent azimuth theoretical resolution [9], [10], but the sub-aperture method introduces aperture division and splicing operation, which increases the complexity of the imaging process. The algorithms based on the full aperture structure are only available for the case of small scanning angle so far [11-13].

In order to make the full aperture imaging algorithms available for the case of large scanning angle, a non-uniform-angular-rate scanning method is proposed for airborne TOPS mode. By adjusting the angular rate in real time, the target of the imaging scene can obtain the same azimuth theoretical resolution.

The main contents of this paper are as follows: Section 2 introduces the TOPS mode imaging geometry and analyzes the problem of inconsistent azimuth theoretical resolution for airborne TOPS mode with large scanning angle. In Sec. 3, by analyzing the Doppler characteristic of the echo in TOPS mode, we establish the equation by which we can adjust the scanning angular rate in real time. In Sec. 4, the proposed beam-scan method is verified with a full aperture imaging frame based on a CS (Chirp Scaling) kernel [13] by simulated data. Section 5 gives the conclusion. 


\section{The Problem of Uniform Beam-scan}

TOPS mode can be assumed to be a constant rotation of the beam centered on a virtual rotation center, as shown in Fig. 1. The coordinate system of the radar imaging plane is XOY in the figure, so the closest distance from the radar to the center of the scene is $r_{0}$, and the closest distance to the virtual rotation center is $r_{\text {rot }}$.

For any point target $P(x, r)$, the instantaneous slant range from the SAR platform to the point target is shown in (1), where $v_{\mathrm{s}}$ is the platform moving speed.

$$
R\left(t_{\mathrm{a}}, r\right)=\sqrt{r^{2}+\left(x-v_{\mathrm{s}} t_{\mathrm{a}}\right)^{2}} .
$$

The phase of the azimuth echo signal is $\Phi_{\mathrm{a}}\left(t_{\mathrm{a}}\right)=$ $-4 \pi R\left(t_{\mathrm{a}}\right) / \lambda, \lambda$ is the wavelength. The first order derivative of the azimuth phase term yields the Doppler frequency, and the expression is expressed by (2), where $\theta_{\mathrm{sq}}$ is the instantaneous observation angle.

$$
f_{\mathrm{a}}=\frac{1}{2 \pi} \frac{\mathrm{d} \Phi_{\mathrm{a}}\left(t_{\mathrm{a}}\right)}{\mathrm{d} t_{\mathrm{a}}}=\frac{2 v_{\mathrm{s}}}{\lambda} \sin \theta_{\mathrm{sq}} .
$$

The Doppler frequency corresponding to the pointing of the beam center is the Doppler centroid frequency, i.e.

$$
f_{\mathrm{ac}}=\left.f_{\mathrm{ac}}\right|_{\theta_{\mathrm{sq}}=\theta_{\mathrm{ac}}}=\frac{2 v_{\mathrm{s}}}{\lambda} \sin \theta_{\mathrm{ac}}
$$

For a SAR mode without beam-scan, the Doppler centroid frequency is a fixed value (regardless of the Doppler center frequency variation with range) for a fixed $\theta_{\mathrm{ac}}$ in (3); but for TOPS mode, the beam scans in a uniform-angular-rate form, that is, $\theta_{\mathrm{ac}}\left(t_{\mathrm{a}}\right)=k_{\varphi} t_{\mathrm{a}}\left(k_{\varphi}\right.$ stands for the beam scanning angular rate, which is a constant in the case of uniform scan) linearly changes with azimuth slow time. Thus, in the TOPS mode, the variation of the Doppler centroid frequency can be expressed in the form of (4), where $K_{\mathrm{c}}$ is the Doppler centroid rate.

$$
K_{\mathrm{c}}=\frac{\mathrm{d}\left[\frac{2 v_{\mathrm{s}}}{\lambda} \sin \theta_{\mathrm{ac}}\left(t_{\mathrm{a}}\right)\right]}{\mathrm{d} t_{\mathrm{a}}}=\frac{2 v_{\mathrm{s}}}{\lambda} k_{\varphi} \cos \left(k_{\varphi} t_{\mathrm{a}}\right) .
$$

When the scanning angle is small, $\cos \left(k_{\varphi} t_{\mathrm{a}}\right) \approx 1$ can be obtained based on the principle of small-angle-approximation, and then the Doppler centroid rate in (4) is a constant, that is, in the TOPS mode with a small scanning angle, the variation of Doppler centroid frequency can be approximated as linear.

We can get the expression of the target Doppler modulation rate by the derivation of the Doppler frequency shown in (2).

$$
K_{\mathrm{a}}=\frac{\mathrm{d} f_{\mathrm{a}}}{\mathrm{d} t_{\mathrm{a}}}=-\frac{2 v_{\mathrm{s}}^{2} \cos ^{3} \theta_{\mathrm{sq}}}{\lambda r} .
$$

Substitute $\theta_{\mathrm{ac}}\left(t_{\mathrm{a}}\right)=k_{\mathrm{c}} t_{\mathrm{a}}$ for $\theta_{\mathrm{sq}}$ in (5), we will get the variation of Doppler modulation rate for the target located

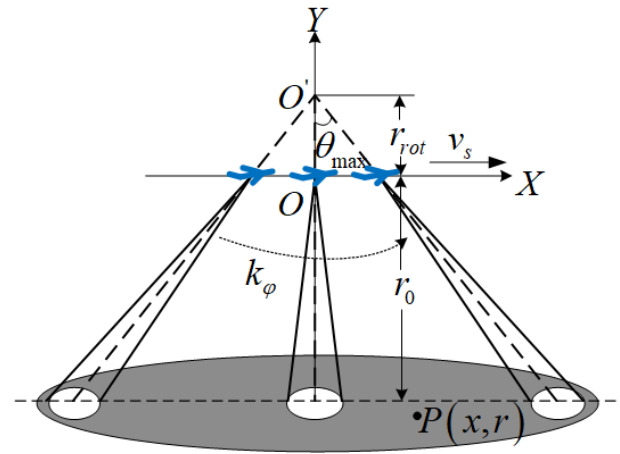

Fig. 1. TOPS mode working principle.

at the beam-center. Similar to the discussion about Doppler centroid frequency before, when the scanning angle is small, the Doppler modulation rate for the target at beamcenter can be approximated as a constant $K_{\mathrm{a}}=-2 v_{\mathrm{s}}{ }^{2} /(\lambda r)$.

For the spaceborne TOPS mode, the maximum scanning angle (shown as $\theta_{\max }$ in Fig. 1) is usually no more than $1^{\circ}$ [5], so we turn to the small-angle-approximation when analyzing the characteristic of azimuth echo, i.e., approximate the target Doppler modulation rate as a constant and the Doppler centroid frequency varies in a linear form. But for the airborne TOPS mode, the scanning angle is usually large to acquire large coverage of the scene, so the small-angle-approximation is no longer valid, which results in two major issue that will directly affect the targets' azimuth resolution: First, since the beam is scanning at a uniform angular rate, the non-uniform-velocity of the beam footprint on the ground must be taken into consideration, which causes the difference in the dwell time for the target with different azimuth location. The second issue is that, as shown in (5), the variation of Doppler modulation rate with the scanning angle needs to be considered when the small-angle-approximation fails. These two issues differ the azimuth theoretical resolution for the targets located at different positions, as the target location is away (in the azimuth direction) from the scene center, the azimuth theoretical resolution degrades, and with larger scanning angle, this problem will become more serious.

\section{A Non-uniform-angular-rate Beam- scan Method}

Aiming at the problem of inconsistent azimuth theoretical resolution exists in uniform-angular-rate TOPS mode with large scanning angle, a non-uniform beam-scan method is proposed in this paper. Firstly, based on the azimuth time-frequency diagram of TOPS mode echo, the relationship between the beam scanning angle and the target Doppler bandwidth is analyzed without the smallangle-approximation. Then, the relationship between the azimuth theoretical resolution of the target and the angular rate of the beam is established. And finally, the values of scanning angular rate that unify the azimuth theoretical resolution of targets with different azimuth locations are calculated. 


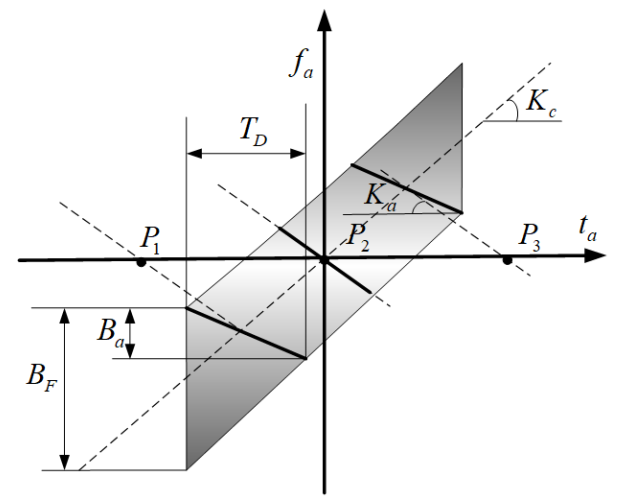

Fig. 2. Azimuth time-frequency diagram of TOPS mode.

Figure 2 shows the azimuth time-frequency diagram of TOPS mode with a large scanning angle. The shaded region in the figure represents the area where the beam sweeps, and the three thick solid lines in the region represent the azimuth time-frequency curves of three points in the scene. The slopes of these lines are different for the fact that the Doppler modulation rate of the target is locationdependent, also shown as a function of scanning angle in (5). In the figure, $T_{\mathrm{D}}$ is dwell time of the beam on a target during the scanning process; $B_{\mathrm{F}}$ stands for the instantaneous Doppler bandwidth of the beam; $B_{\mathrm{a}}$ is the Doppler bandwidth of the target in the TOPS mode, $K_{\mathrm{a}}$ is the Doppler modulation rate of the target in the TOPS mode and $K_{\mathrm{c}}$ stands for Doppler centroid rate. Taking the point target $P_{1}$ in the scene as an example, the expression of the dwell time $T_{\mathrm{D}}$ can be obtained by (6).

$$
T_{\mathrm{D}}=\frac{B_{\mathrm{F}}}{K_{\mathrm{c}}+\left|K_{\mathrm{a}}\right|} \text {. }
$$

Combining (4), (5) and (6), we can obtain the relationship between the target Doppler bandwidth and the beam instantaneous Doppler bandwidth.

$$
B_{\mathrm{a}}=\left[1+\frac{k_{\varphi} r}{v_{\mathrm{s}} \cos ^{2} \theta_{\mathrm{ac}}\left(t_{\mathrm{a}}\right)}\right]^{-1} B_{\mathrm{F}} .
$$

The beam instantaneous Doppler bandwidth $B_{\mathrm{F}}$ is also equal to the Doppler bandwidth of the target in the stripmap mode. Therefore, the shrinking factor in the TOPS mode with large scanning angle as shown in (8) is defined:

$$
\alpha\left(t_{\mathrm{a}}\right)=1+\frac{k_{\varphi} r}{v_{\mathrm{s}} \cos ^{2} \theta_{\mathrm{ac}}\left(t_{\mathrm{a}}\right)} .
$$

It is not difficult to find that in the case where the beam scanning angle $\theta_{\mathrm{ac}}\left(t_{\mathrm{a}}\right)$ is relatively large, the value of $\alpha$ changes with the scanning angle and thus is a function of azimuth slow time $t_{\mathrm{a}}$. When the scanning angle is small, we can get $\cos ^{2} \theta_{\mathrm{ac}}\left(t_{\mathrm{a}}\right) \approx 1$ based on the principle of small-angle-approximation, and the shrinking factor is degenerated to a constant one defined in the traditional uniform-angular-rate beam-scan TOPS mode [4].
Since the shrinking factor can be used to establish the relationship of azimuth theoretical resolution between TOPS mode and stripmap mode, the azimuth theoretical resolution of point target in the TOPS mode can be expressed as

$$
\rho_{\mathrm{a}, \text { tops }}=\alpha \cdot \rho_{\mathrm{a} \text {,strip }} \cdot
$$

From (8), we can see that in the case of large scanning angle, the shrinking factor changes with the azimuth slow time, and in order to make the azimuth theoretical resolution of the point target in the scene to be a constant, let the beam scanning angular rate in (8) also changes with azimuth slow time, that is, unify the azimuth theoretical resolution by adjusting the beam scanning angular rate in real time, which is the idea of this paper.

First, establish the relationship between the beam scanning angle and the scanning angular rate, which is shown in (10).

$$
\theta_{\mathrm{ac}}\left(t_{\mathrm{a}}\right)=\int_{0}^{t_{\mathrm{a}}} k_{\varphi}(\xi) \mathrm{d} \xi .
$$

By combining (8) and (10), (9) can be further written in the form of (11)

$$
\rho_{\mathrm{a}, \text { tops }}=\left(1+\frac{r_{0} k_{\varphi}\left(t_{\mathrm{a}}\right)}{v_{\mathrm{s}}\left(\cos \left(\int_{0}^{t_{\mathrm{a}}} k_{\varphi}(\xi) \mathrm{d} \xi\right)\right)^{2}}\right) \cdot \rho_{\mathrm{a}, \text { strip }} .
$$

It can be seen that (11) cannot obtain explicit solution, so we can approximate a recursive solution to (11) based on the idea of differential. Discretize the azimuth slow time, that is, $t_{\mathrm{a}}(n)=n \cdot \Delta T, n=-N / 2, \ldots, 0, \ldots, N / 2$ where $\Delta T$ stands for the azimuth time sampling interval, $N$ is azimuth sampling point during a complete beam scanning process. In order to be able to recursively calculate the beam rotation angular rate for each azimuth slow time, $\cos \theta\left(t_{\mathrm{a}}\right)$ is approximated as in (12).

$$
\cos \theta\left(t_{\mathrm{a}}\right) \approx \cos \left(\int_{0}^{(n-1) \Delta T} k_{\varphi}(\xi) \mathrm{d} \xi\right) .
$$

Substitute (12) into the (11), we will obtain (13) by which we can recursively calculate the beam scanning angular rate for each azimuth slow time, during the calculation, let $\rho_{\mathrm{a} \text {, tops }}$ and $\rho_{\mathrm{a} \text {, strip }}$ remain unchanged (the initial values of them are determined by system parameters at the azimuth scene center), which obeys the requirement of azimuth theoretical resolution consistency.

$$
k_{\varphi}\left[t_{\mathrm{a}}(n)\right]=\frac{v_{\mathrm{s}}}{r_{0}}\left(\frac{\rho_{\mathrm{a}, \text { tops }}}{\rho_{\mathrm{a} \text {, strip }}}-1\right) \cdot\left\{\cos \left[\int_{0}^{(n-1) \Delta T} k_{\varphi}(\xi) \mathrm{d} \xi\right]\right\}^{2} .
$$

Figure 3 shows how the scanning angular rate changes with azimuth slow time. The parameters used in the simulation are shown in Tab. 1. 


\begin{tabular}{|c|c|c|c|}
\hline Carrier Frequency & $10 \mathrm{GHz}$ & PRF & $2000 \mathrm{~Hz}$ \\
\hline Bandwidth & $60 \mathrm{MHz}$ & Azimuth Aperture & $0.1 \mathrm{~m}$ \\
\hline $\begin{array}{c}\text { Closest Slant Range } \\
\text { of Scene Center }\end{array}$ & $10 \mathrm{~km}$ & Platform Velocity & $50 \mathrm{~m} / \mathrm{s}$ \\
\hline Platform Altitude & $5 \mathrm{~km}$ & $\begin{array}{c}\text { Azimuth Beam } \\
\text { Width }\end{array}$ & $15.59^{\circ}$ \\
\hline $\begin{array}{c}\text { Maximum Scanning } \\
\text { Angle }\end{array}$ & $20^{\circ}$ & $\begin{array}{c}\text { Azimuth } \\
\text { Resolution }\end{array}$ & $5 \mathrm{~m}$ \\
\hline
\end{tabular}

Tab. 1. System working parameters.

It can be seen from Fig. 3 that in order to maintain the consistency of the target azimuth theoretical resolution in the azimuth scene, the angular rate of the non-uniform scanning method is gradually reduced as the scanning angle increases.

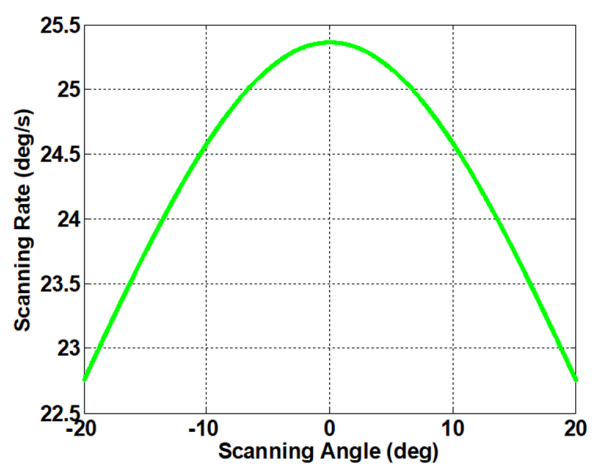

Fig. 3. Variation of angular rate in non-uniform beam-scan method.

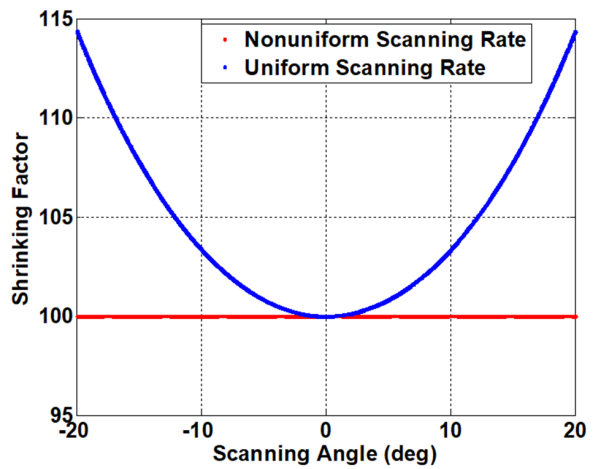

(a)

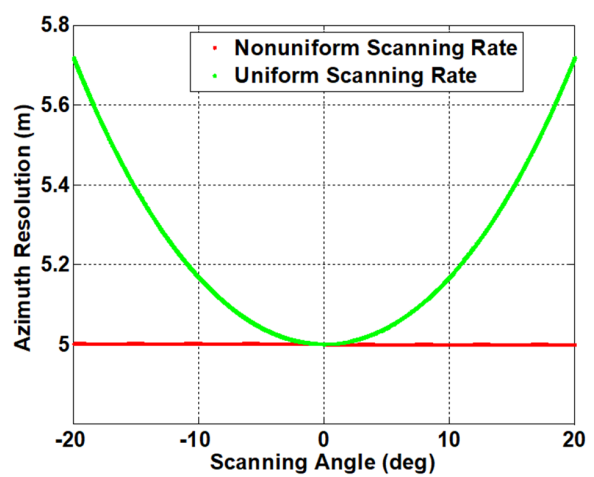

(b)

Fig. 4. Variation of (a) shrinking factor and (b) azimuth resolution in both uniform and non-uniform beam-scan method.
Substitute the calculated angular rate values into (8) and (9), we can observe how shrinking factor and azimuth theoretical resolution change in the non-uniform-angularrate beam-scan method, we draw the curves in Fig. 4 together with the uniform-angular-rate beam-scan method as a comparison.

It can be seen from Fig. 4 that for the TOPS mode with uniform-angular-rate beam-scan, when the maximum scanning angle is $20^{\circ}$, the azimuth theoretical resolution of the target near the edge of azimuth imaging scene is increased by $0.7 \mathrm{~m}$ compared with the target at the scene center, and it is conceivable that when the maximum scanning angle is increased, the azimuth theoretical resolution of the target at the edge of the scene will further deteriorate. However, the shrinking factor and the azimuth theoretical resolution are constant in the non-uniform-angularrate beam-scan method, which has theoretically verified that the scanning angular rate calculated by (13) can maintain the consistency of the azimuth theoretical resolution in the scene.

\section{Simulation Analyses}

In order to further verify the validity of the non-uniform beam scanning method proposed in this paper, a set of point targets irradiated by TOPS mode with non-uniform-angular-rate beam-scan is simulated. The range interval of targets is $400 \mathrm{~m}$ and the azimuth interval is $750 \mathrm{~m}$, the system parameters are listed in Tab. 1. In order to verify the method in an efficient way, we choose the full aperture imaging frame proposed in reference [13], in which the Doppler aliasing is first removed by an azimuth preprocess, then realize the range dimension process based on the CS method and at last finish the azimuth compression by azimuth scaling with SPECAN (SPECtral ANalysis) method. The final imaging results are shown in Fig. 5.

Three point targets at the range center with different azimuth positions are selected from Fig. 5, and their azimuth profiles are drawn in Fig. 6.

In order to quantitatively analyze the focus of targets at different azimuth positions both in the case of uniform and non-uniform beam-scan, the two-dimensional resolu-

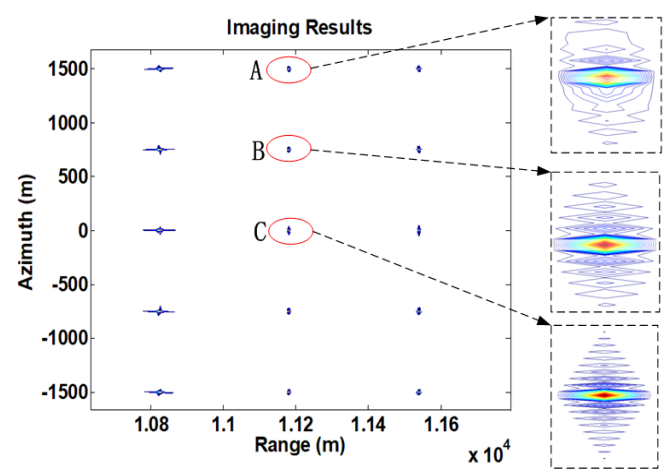

Fig. 5. Imaging results of TOPS with non-uniform-angularrate beam-scan. 

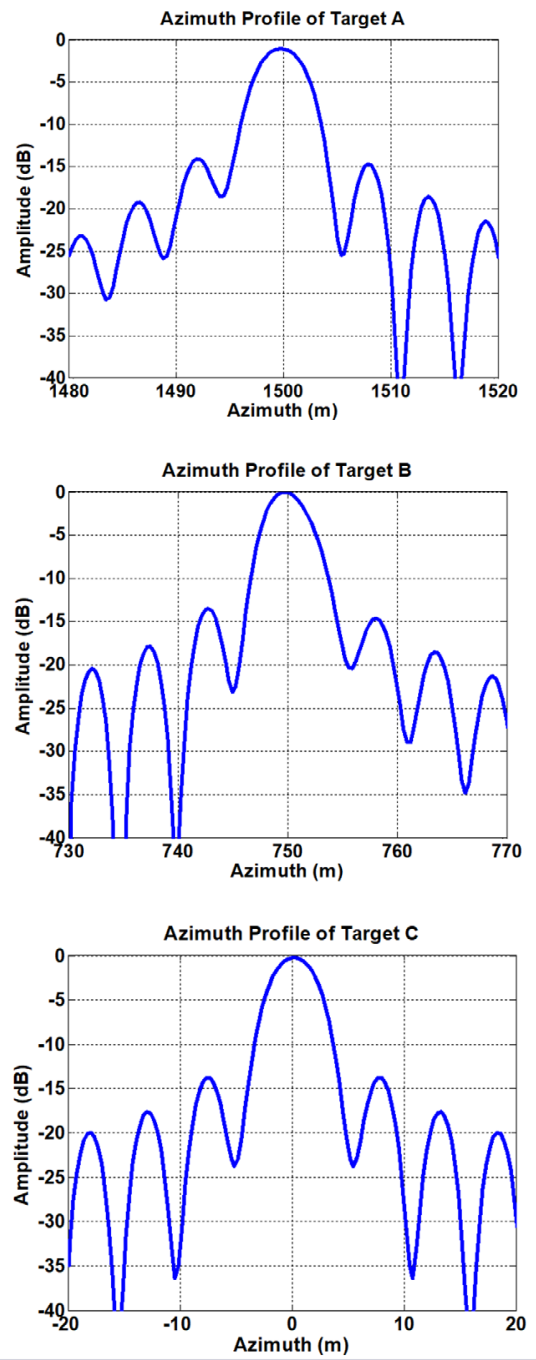

Fig. 6. Azimuth profiles of the selected targets.

\begin{tabular}{|c|c|c|c|c|c|}
\hline \multirow{2}{*}{$\begin{array}{c}\text { Scan } \\
\text { method }\end{array}$} & \multirow{2}{*}{ Target } & \multicolumn{2}{|c|}{ Azimuth } & \multicolumn{2}{c|}{ Range } \\
\cline { 3 - 6 } & & $\begin{array}{c}\text { PSLR } \\
(\mathbf{d B})\end{array}$ & $\begin{array}{c}\text { Resolution } \\
(\mathbf{m})\end{array}$ & $\begin{array}{c}\text { PSLR } \\
(\mathbf{d B})\end{array}$ & $\begin{array}{c}\text { Resolution } \\
(\mathbf{m})\end{array}$ \\
\hline \multirow{3}{*}{ Uniform } & $\mathbf{A}$ & -12.9 & 5.44 & -13.0 & 5.55 \\
\cline { 2 - 6 } & $\mathbf{B}$ & -13.2 & 5.25 & -13.0 & 5.21 \\
\cline { 2 - 6 } & $\mathbf{C}$ & -13.2 & 5.01 & -12.9 & 5.01 \\
\hline \multirow{3}{*}{$\begin{array}{c}\text { Non- } \\
\text { uniform }\end{array}$} & $\mathbf{A}$ & -13.0 & 5.02 & -13.1 & 5.49 \\
\cline { 2 - 6 } & $\mathbf{B}$ & -13.1 & 5.02 & -13.0 & 5.23 \\
\cline { 2 - 6 } & $\mathbf{C}$ & -13.1 & 5.01 & -12.9 & 4.99 \\
\hline
\end{tabular}

Tab. 2. Quantitative analysis of focus in both methods.

tion and the PSLR (Peak Side Lobe Ratio) in the two cases are calculated, and the results are shown in Tab. 2.

It can be seen from Tab. 2 that the non-uniform-angular-rate scanning method can improve the azimuth resolution of the targets near the edge of azimuth scene in relative to the traditional uniform-angular-rate scanning.

However, the non-uniform-beam scan method brings the problem of worse linearity of Doppler centroid frequency, which can be seen in Fig. 7.

In Fig. 7, the red and blue curves stand for non-uniform and uniform beam-scan method respectively, and the green curve means a constant Doppler centroid rate. It can

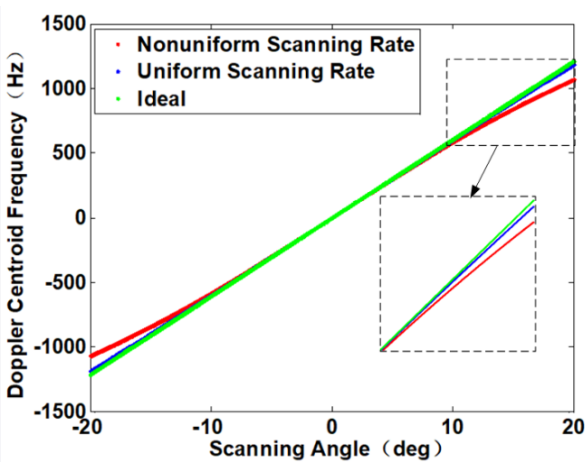

Fig. 7. Variation curves of Doppler centroid frequency.

be seen that the non-uniform beam-scan method results in worse linearity of Doppler centroid frequency than uniform beam-scan method. The problem of worse linearity of Doppler centroid frequency is that, for most full-aperture algorithms, the accuracy of these algorithms depends on the linearity of Doppler centroid frequency, and thus the constant Doppler centroid rate shown as the green curve in the figure is ideal for these algorithms. On the contrary, the worse the linearity of Doppler centroid frequency, the worse the accuracy is of these full aperture algorithms.

For the spaceborne TOPS mode, the worse linearity of Doppler centroid frequency may result in evident degradation of azimuth resolution and thus is an issue must be tackled. For the airborne TOPS mode, e.g., with the system parameters used in Tab. 1, the effect of the worse linearity of Doppler centroid frequency to the degradation of azimuth resolution is about $0.02 \mathrm{~m}$ (as shown in Tab. 2) when the theoretical azimuth resolution is $5 \mathrm{~m}$, and thus the issue is treated negligible in the paper.

\section{Conclusions}

This paper discusses the problem of inconsistency of the azimuth theoretical resolution in airborne TOPS mode with a large scanning angle. By analyzing the relationship of azimuth theoretical resolution and beam scanning angular rate, we propose a non-uniform-angular-rate beam-scan method. The effectiveness of the method is verified by simulation. Further discussion of the worse linearity of Doppler centroid frequency brought by non-uniform beamscan will be left as future work.

\section{References}

[1] CERUTTI-MAORI, D., KLARE, J., BRENNER, A. R., et al. Wide-area traffic monitoring with the SAR/GMTI system PAMIR. IEEE Transactions on Geoscience and Remote Sensing, 2008, vol. 46, no. 10, p. 3019-3030. DOI: 10.1109/TGRS.2008.923026

[2] BRENNER, A. R., ENDER, J. H. G. Demonstration of advanced reconnaissance techniques with the airborne SAR/GMTI sensor PAMIR. IEE Proceedings - Radar, Sonar and Navigation, 2006, vol. 153, no. 2, p. 152-162. DOI: 10.1049/ip-rsn:20050044 
[3] VAN DER GRAAF, M. W., OTTEN, M. P. G., HUIZING, A. G., et al. AMBER: An X-band FMCW digital beam forming synthetic aperture radar for a tactical UAV. In IEEE International Symposium on Phased Array Systems and Technology. Waltham (MA, USA), 2013, p. 165-170. DOI: 10.1109/ARRAY.2013.6731820

[4] DE ZAN, F., MONTI GUARNIERI, A. TOPSAR: Terrain observation by progressive scans. IEEE Transactions on Geoscience and Remote Sensing, 2006, vol. 44, no. 9, p. 2352-2360. DOI: 10.1109/TGRS.2006.873853

[5] META, A., MITTERMAYER, J., PRATS, P., et al. TOPS imaging with TerraSAR-X: Mode design and performance analysis. IEEE Transactions on Geoscience and Remote Sensing, 2010, vol. 48, no. 2, p. 759-769. DOI: 10.1109/TGRS.2009.2026743

[6] WU, Y. F., SUN, G. C., XIA, X. X., et al. An azimuth frequency non-linear chirp scaling (FNCS) algorithm for TOPS SAR imaging with high squint angle. IEEE Journal of Selected Topics in Applied Earth Observation and Remote Sensing, 2014, vol. 7, no. 1, p. 213-221. DOI: 10.1109/JSTARS.2013.2258893

[7] SUN, G. C., XING, M. D., WANG, Y., et al. Sliding spotlight and TOPS SAR data processing without subaperture. IEEE Geoscience and Remote Sensing Letters, 2011, vol. 8, no. 6, p. 1036-1040. DOI: $10.1109 /$ LGRS.2011.2151174

[8] PRATS, P., SCHEIBER, R., MITTERMAYER, J., et al. Processing of sliding spotlight and TOPS SAR data using baseband azimuth scaling. IEEE Transactions on Geoscience and Remote Sensing, 2010, vol. 48, no. 2, p. 770-780. DOI: 10.1109/TGRS.2009.2027701

[9] XU, W., HUANG, P. P., DENG, Y. K., et al. An efficient approach with scaling factors for TOPS-mode SAR data focusing. IEEE Geoscience and Remote Sensing Letters, 2011, vol. 8, no. 2 , p. 929-933. DOI: 10.1109/LGRS.2011.2135837

[10] XU, W., HUANG, P. P., WANG, R., et al. TOPS-mode raw data processing using chirp scaling algorithm. IEEE Journal of Selected Topics in Applied Earth Observation and Remote Sensing, 2014, vol. 7, no. 1, p. 235-246. DOI: 10.1109/JSTARS.2013.2260134
[11] YANG, J., SUN, G. C., XING, M., et al. Squinted TOPS SAR imaging based on modified range migration algorithm and spectral analysis. IEEE Geoscience and Remote Sensing Letters, 2014, vol. 11, no. 10, p. 1707-1711. DOI: 10.1109/LGRS.2014.2306433

[12] SUN, G. C., XING, M., XIA, X. G., et al. Beam steering SAR data processing by a generalized PFA. IEEE Transactions on Geoscience and Remote Sensing, 2013, vol. 51, no. 8, p. 4366-4377. DOI: 10.1109/TGRS.2012.2237407

[13] YANG, W., LI, C. S., CHEN, J., et al. A novel three-step focusing algorithm for TOPSAR image formation. In Proceedings of the 2010 IEEE International Geoscience and Remote Sensing Symposium (IGARSS). Honolulu (HI, USA), 2010, p. 4087-4090. DOI: $10.1109 /$ IGARSS.2010.5649954

\section{About the Authors ...}

Yifu GUAN (corresponding author) was born in Harbin, Heilongjiang Province, China, in 1988. He received the BS and $\mathrm{MSc}$ degree in Electronic Intelligence from the Aviation University of Air force, Changchun, China, in 2012 and 2015, respectively. He is currently working toward the Ph.D. degree at the National University of Defense Technology of China. His research interests include radar imaging algorithms, FMCW SAR, and real-time processing.

Wenge CHANG received the BS, MSc, and Ph.D. degrees in Information and Communication Engineering from the National University of Defense Technology, Changsha, China, in 1987, 1993, and 2001, respectively. He is currently a professor with the National University of Defense Technology. His fields of interest include synthetic aperture radar system, real-time signal processing and SAR image processing. 